\title{
Full-Field Strain Analysis of Porous Membrane Under Biaxial Tension
}

\author{
Wang Wei ${ }^{1}$, Xu Xiaochen ${ }^{2}$, Xu Zhihong ${ }^{1}$ \\ ${ }^{1}$ Department of Civil Engineering, Nanjing University of Science and Technology, Nanjing, China \\ ${ }^{2}$ Nanjing Institute of Electronic Technology, Nanjing, China
}

\section{Email address:}

122191841@qq.com (Wang Wei)

\section{To cite this article:}

Wang Wei, Xu Xiaochen, Xu Zhihong. Full-Field Strain Analysis of Porous Membrane Under Biaxial Tension. Science Discovery. Vol. 7, No. 2, 2019, pp. 107-114. doi: 10.11648/j.sd.20190702.19

Received: March 25, 2019; Accepted: March 20, 2019; Published: May 23, 2019

\begin{abstract}
In this paper, the full-field strain and strain concentration factor around the hole of PTFE membrane under uniaxial biaxial tension are studied based on digital image speckle (DIC) technique and finite element calculation.Through the load-strain curve of porous membrane during tension, three stages of properties of membrane during biaxial tension are obtained: elastic stage, plastic stage and failure stage.The relationship between the maximum strain around the pore, the ultimate bearing capacity of the membrane and the pore size is obtained. The relationship between the strain concentration factor at the pore edge and the pore size is obtained.The results show that the larger the pore size, the more obvious the strain concentration around the pore and the smaller the ultimate load it can bear, and the greater the strain concentration coefficient around the pore, the more easily the membrane is damaged.
\end{abstract}

Keywords: Digital Image, Finite Element, Strain, Aperture

\section{含孔膜材双向拉伸下断裂全场应变分析}

\section{王伟 ${ }^{1}$, 徐晓晨 ${ }^{2}$, 徐志洪 ${ }^{1}$}

1南京理工大学理学院土木工程系, 南京, 中国

2南京电子技术研究所, 南京, 中国

\section{邮箱}

122191841@qq.com (王伟)

摘要：该文基于数字图像散斑（DIC）技术及有限元计算对比研究了含孔PTFE膜材在单轴双向拉伸下的全场应变以及 孔周围的应变集中系数。通过含孔膜材拉伸过程荷载--孔周最大应变曲线得到膜材在双向拉伸过程中呈现出了三阶段 性质：弹性阶段、塑性阶段以及破坏阶段; 得到了含孔膜材断裂时孔周的最大应变、膜材的极限承载力与孔径大小之 间的关系, 分析得到了膜材孔边应变集中系数与孔径之间的关系。结论表明: 孔径越大, 其孔周应变集中现象越明显, 所能承受的极限荷载越小; 且孔边应变集中系数越大，膜材越易损坏。

关键词: 数字图像, 有限元, 应变, 孔径 


\section{1. 引言}

建筑膜结构是 20 世纪中期出现的新型空间结构体系 [1], 膜材料具有强度高、柔软度好等特征[2], 但膜结构 在安装[3]和长期使用的过程中因诸多原因造成膜面局部 出现微小裂口甚至孔洞, 在膜结构张拉状态下, 就会在膜 材孔洞周边产生应力集中现象并逐渐扩展延伸，最终致使 膜结构膜面破坏, 引发事故。因此, 对含裂缝或含孔膜材 的力学性能的研究就显得非常有必要。

近年来, 已有相关国内外学者对建筑膜材料进行了各 种力学试验探究以及数值模拟。如D.Bigaud等[4]对聚氯乙 烯膜材料的裂纹扩展行为进行了研究, 得到PVC膜材的撕 裂破坏机理, PVC膜材的裂纹扩展往往由裂纹尖端展开, 在单轴受拉状态下, 无论膜材料初始裂缝位于何方向, 其 裂纹必然会沿垂直于荷载拉伸的方向扩展。中国矿业大学 张营营等[5-6]研究了不同试验条件下PTFE膜材料的力学 性能, 并对其破坏机理、抗力不定性分析以及黏弹性本构 关系进行了探讨，得知PTFE膜材料的黏弹性性能与初始 应力的关系较为密切, 而受初始拉伸速率影响较小。东华 大学马倩[7]在对无损膜材撕裂破坏机理的数值模拟中提 出机织物的撕裂破坏由应力集中造成, 膜材的撕裂过程主 要由三步构成: 受拉伸系统纱线受力伸直, 屈曲消失; 非 受拉伸系统纱线的横向滑移, 剪切; 非受拉伸系统纱线的 拉伸破坏。目前的已有研究多数基于无损膜材的各项力学 性能, 对于损伤膜材受拉状态下的应变测量也多数基于传 统的贴应变片或引伸计测量方法, 但由于膜材料表面的不 平整性且受力变形的繁杂性, 该方法很难精确的测出其真 实变形以及全场应变情况。

本文结合数字图像散斑技术（DIC）[8-9]和有限元计 算分析对比了PTFE膜材在含孔情况下受拉时的全场应 变, 孔周最大应变以及应变集中系数, 以期为膜结构的安 全评估、修复与健康监测 [10]提供一定的理论基础。

\section{2. 实验材料与方法}

\section{1. 实验材料及仪器}

本文所选实验材料为PTFE建筑用膜，该膜材中间层 纤维为玻璃纤维丝通过平纹法[11]交织在一起构成膜材的 基布, 膜材表面涂覆一层特氟龙树脂, 加工形成该种编织 的柔性材料。为确保实验结果的稳定可靠与可比性, 本实 验所用材料均为同一批次生产的PTFE膜材, 其具体参数 如表1所示。

本实验对含不同孔径PTFE膜材进行单轴双向拉伸下 的断裂破坏, 所用实验仪器为 (1) 本研究室自主研发的 双轴万能拉伸试验机如图1; (2) DIC 数字图像测量系统 如图2。

DIC 数字图像测量系统实验装置包括以下 3 个部分: 数 字图像收集系统、数字图像分析处理系统以及照明系统。
一般测量步骤为CCD相机采集实验对象散斑图像后, 将图 像导入到计算机内的光学测量变形软件 GOM Correlate Professional计算分析, 得到实验对象的变形及应变等数据 结果, 其非接触式测试[12]步骤示意图如图3所示。

表1 PTFE膜材具体参数。

\begin{tabular}{llll}
\hline 项目 & 单位 & 保证值 & 试验值 \\
\hline 膜材质量 & $\mathrm{g} / \mathrm{m}^{2}$ & $1300 \pm 130$ & 1272 \\
基材质量 & $\mathrm{g} / \mathrm{m}^{2}$ & $500 \pm 50$ & 508 \\
涂层质量 & $\mathrm{g} / \mathrm{m}^{2}$ & $800 \pm 80$ & 764 \\
膜材厚度 & $\mathrm{mm}$ & $0.80 \pm 0.10$ & 0.70 \\
& 经向 & $25+2 /-1$ & 26 \\
编织密度 & $\mathrm{pcs} / 2.5 \mathrm{~cm}$ & & \\
& 纬向 & $19+2 /-1$ & 20 \\
& 经向 & $1500 \pm 150$ & 1545 \\
弹性模量 & $\mathrm{MPa}$ & & \\
& 纬向 & $850 \pm 80$ & 881 \\
\hline
\end{tabular}

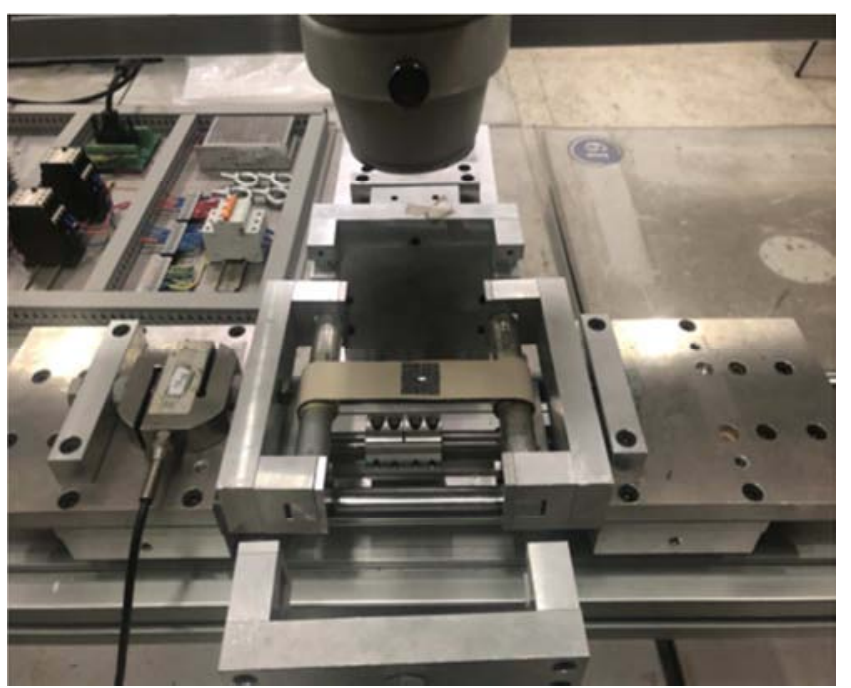

图1 双轴万能拉伸试验机。

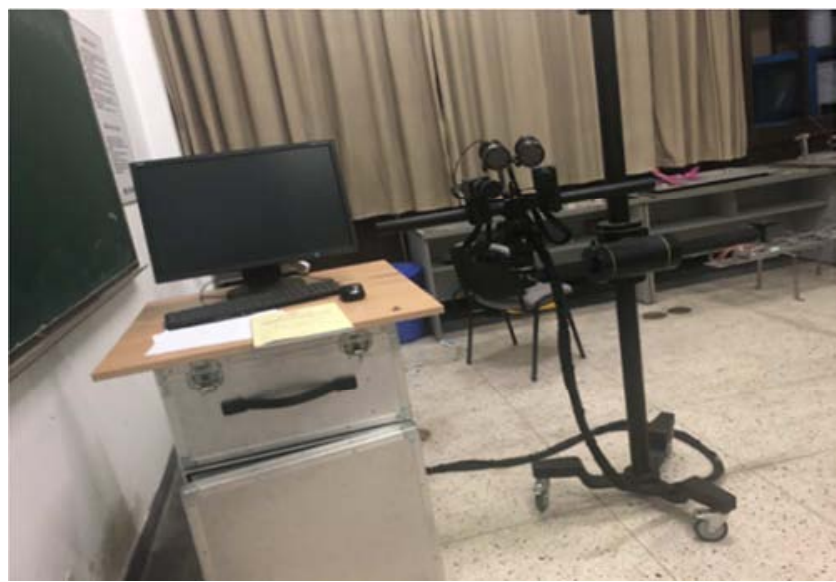

图2 DIC数字图像测量系统。 


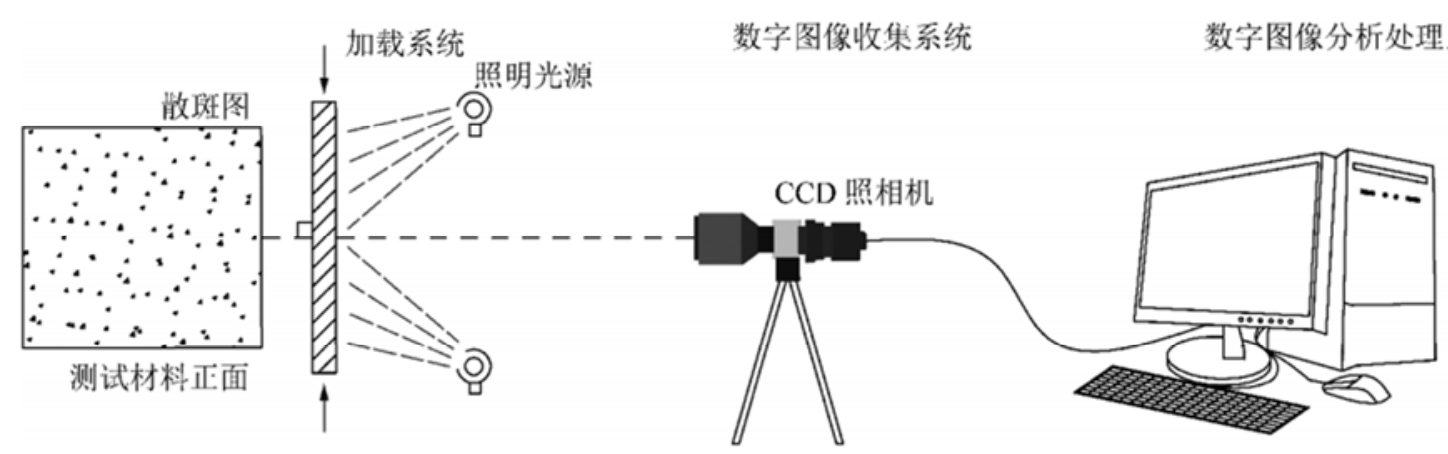

图3 非接触式测试步骤示意图。

\section{2. 实验方案及步骤}

本文主要研究含不同孔径PTFE膜材在单轴双向拉伸 直至断裂下的力学特性, 所选用PTFE膜材拉伸试样尺寸 为 $500 \mathrm{~mm} \times 30 \mathrm{~mm}$, 制斑区域为膜材中间部位 $100 \mathrm{~mm} \times 30 \mathrm{~mm}$ 处, 两端 $200 \mathrm{~mm} \times 30 \mathrm{~mm}$ 部位用于缠绕试验 机夹具, 以便膜材后期的拉伸, 膜材实物图及示意图如图
4所示。在膜材试样的中心位置打孔, 孔径分别为 $2 \mathrm{~mm}$ 和 $4 \mathrm{~mm}$, 膜材打孔采用机械钻孔方式[13]; 为了采用DIC方 法测量膜材拉伸时的变形, 需要在试样上制作散斑, 制斑 方法选用MATLAB设计的散斑图打印到水转印膜上,而后 直接将水转印膜纹到各组膜材表面制成散斑图。
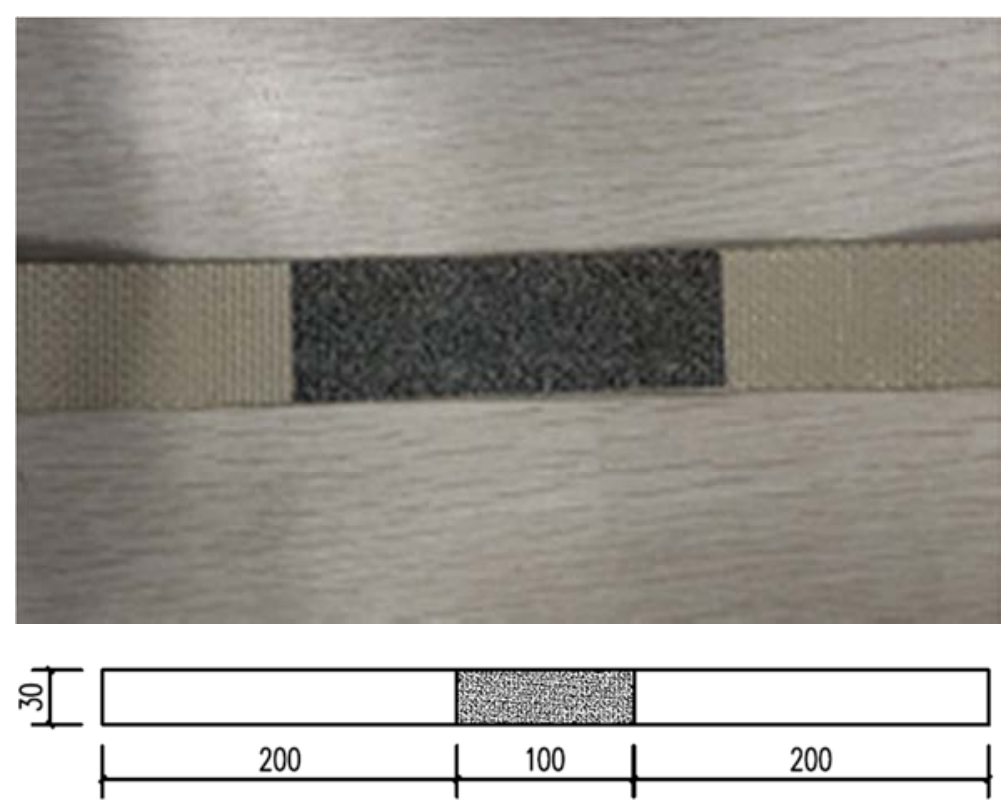

图4 含散斑膜材实物图及CAD图。

本次双向拉伸试验具体步骤为: 1) 将X方向两端缠绕 式夹具与双轴万能拉伸试验机运动台面用螺丝固定, 其中 一端的缠绕式夹具的一侧与力传感器固定一侧与膜材直 接相连, 由于在拉伸试验中缠绕式夹具的钳口会对试验结 果产生重要影响[14], 故在将膜材缠绕进夹具之前先将夹 口钝化处理, 以避免在拉伸过程中夹口处膜材产生应力集 中断裂而导致实验失败, 另一端的缠绕式夹具同样与膜材 直接相连；2）膜材安装好以后, 首先对其施加预张力, 预加张力是为了消除试样在加载初始状态时的松弛现象, 本实验采用的预加张力为 $10 \mathrm{~N} ; 3$ ) 启动双轴万能拉伸试验 机, 对PTFE膜材进行双向拉伸, 数字图像测量系统每隔 $1 \mathrm{~s}$ 自动采集一张膜材拉伸过程中的散斑图像直至膜材拉 伸断裂停止采集同时停止试验机运行；4）将各组实验采
集到的散斑图像导入计算机 DIC 测试系统的 GOM Correlate Professional分析软件进行双向拉伸下直至膜材 断裂的力学特性研究。

\section{3. 实验结果与分析}

\section{1. 含孔膜材全场应变}

为了更加直观地了解含孔膜材在拉伸过程直至断裂 的全场应变, 两组孔径同时选取测力点为 $1 \mathrm{KN} 、 2 \mathrm{KN}$ 、 $3 \mathrm{KN} 、 4 \mathrm{KN}$ 以及各自对应的断裂峰值力所对应的散斑图进 行分析, 分别导出其对应的应变场如图5所示。 

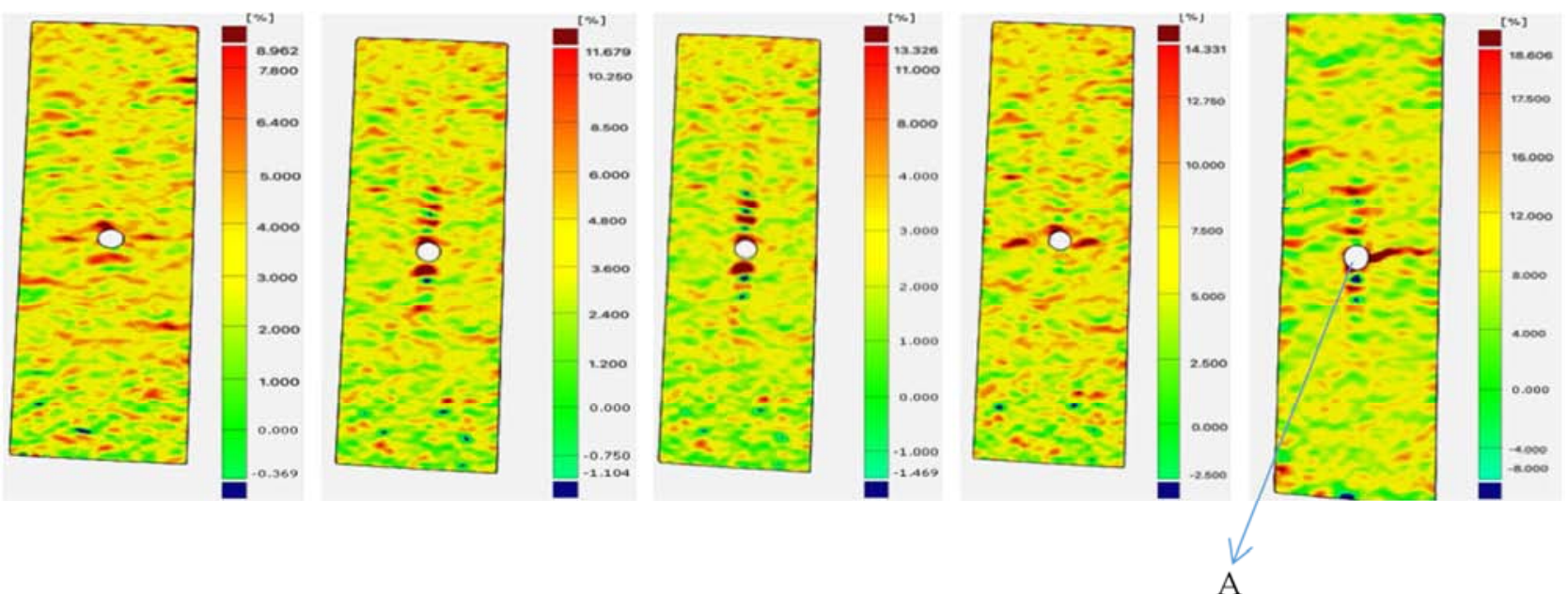

(a)中心 $2 \mathrm{~mm}$ 孔应变云图演变
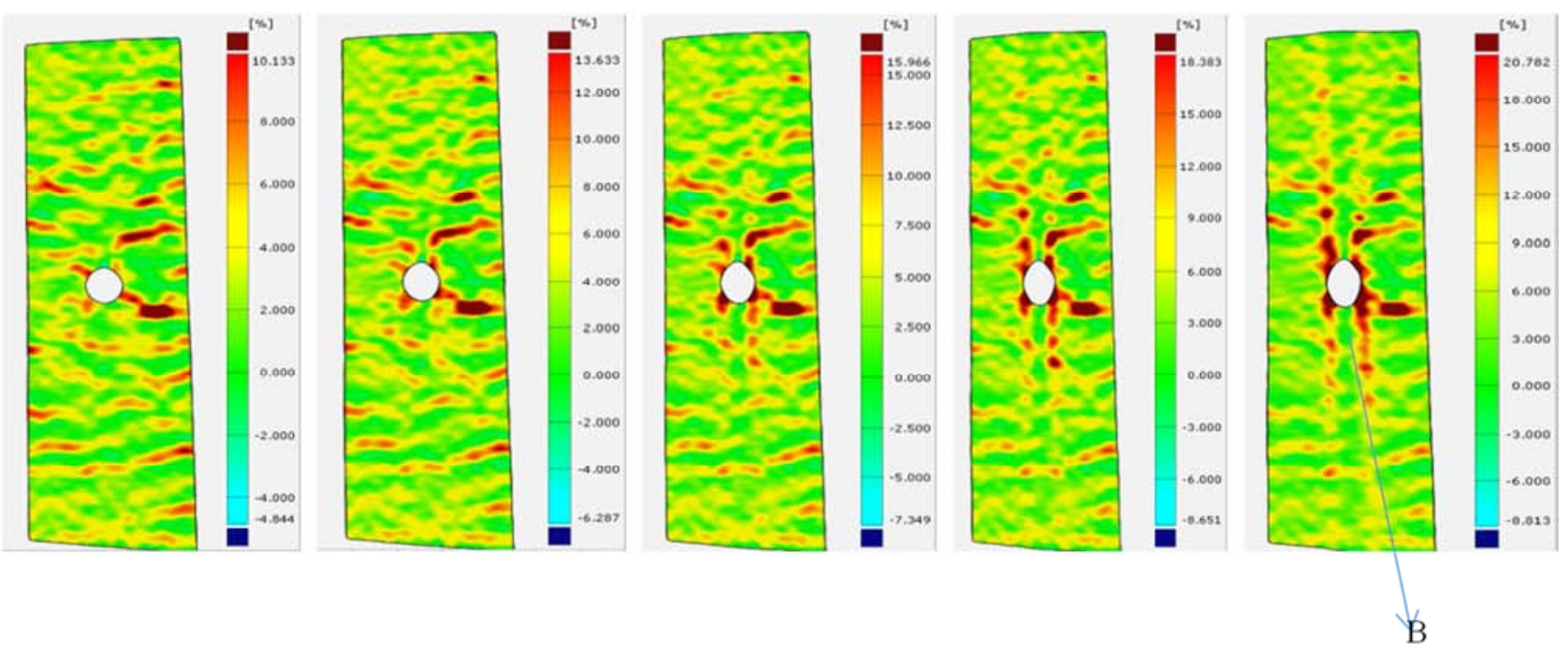

(b)中心 $4 \mathrm{~mm}$ 孔应变云图演变

图5 双向拉伸下孔边应变场演变云图。

由图5所示双向拉伸下含孔膜材应变场演变云图可以得 知, 在含孔膜材的初始拉伸阶段, 孔周边均出现了应变集中 现象, 膜材的上下部位应变交替分布, 这说明由于PTFE膜 材孔的存在, 从膜材开始受力时, 圆孔就已经对整张膜材产 生了不可忽视的影响, 由于膜材为各向正交异性材料, 故在 膜材的不同区域均出现了小范围的应变集中带, 说明膜材集 中分布的随机性、弥散性。随着荷载的持续增加, 膜材孔周 围应变集中现象更为明显，含 $2 \mathrm{~mm}$ 孔膜材断裂时应变达到 $18.606 \%$, 且沿着膜材横向出现一条极其明显的应变集中带, 由孔心指向膜的侧边, 则膜材最终会沿着该应变集中带发生 撕裂, 而在远离孔的上下部位, 随着荷载的加大膜材该部位 不再像加载初始阶段出现应变集中, 说明离孔越远, 荷载越 大时, 受孔的集中应变影响的效益会减弱; 含 $4 \mathrm{~mm}$ 孔膜材最 大应变达到 $20.762 \%$, 同样从膜材孔心出发向两边延伸直至 膜材破坏。而对于 $2 \mathrm{~mm}$ 孔膜材以及 $4 \mathrm{~mm}$ 孔膜材在荷载持续加 大拉伸过程中均出现了膜材局部收缩的现象, 这是由于膜材 为编织材料, 纬向纱线在张紧伸长过程中导致膜材经向纱线 发生褶皱而收缩。为了分析膜材中心含不同直径孔在整个拉 伸过程中孔周最大应变 (即如图 5 所示 $2 \mathrm{~mm}$ 孔膜材 $\mathrm{A}$ 点以及
$4 \mathrm{~mm}$ 孔膜材 B 点) 的情况, 每200N选取一张散斑图, 根据DIC 计算软件数据, 将这两种孔径膜材在拉伸过程中的应变变化 绘制成图6所示图线。

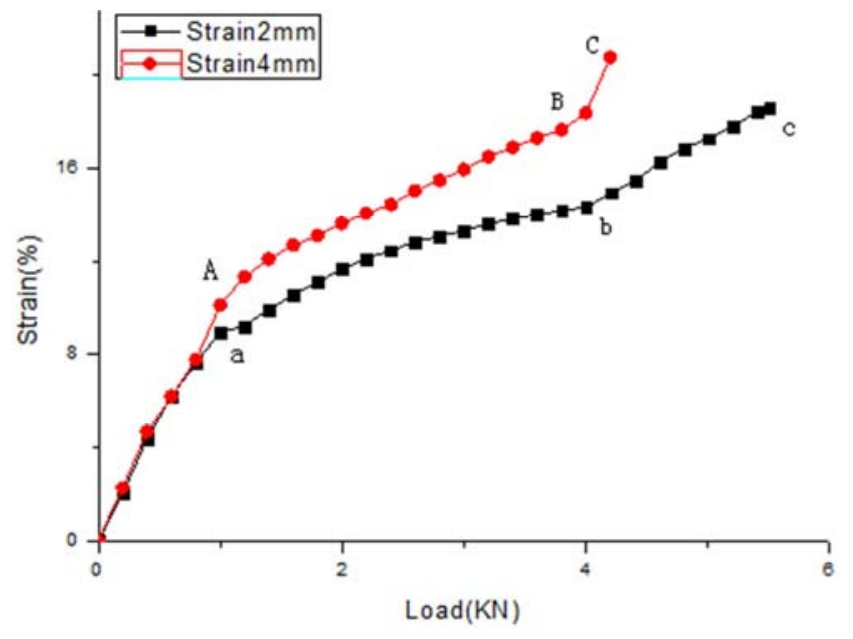

图6 膜材双向拉伸下最大应变场指标演化图。 
由上图可以看出, 两种孔径膜材均在双向拉伸荷载持 续增加过程中出现了三个阶段, 即弹性阶段 (中心 $2 \mathrm{~mm}$ 孔 Oa阶段，4mm孔OA阶段）、塑性阶段（中心 $2 \mathrm{~mm}$ 孔 $\mathrm{mb}$ 阶 段, $4 \mathrm{~mm}$ 孔A阶段) 以及破坏阶段 (中心 $2 \mathrm{~mm}$ 孔bc阶段, $4 \mathrm{~mm}$ 孔BC阶段）。所谓弹性阶段就是PTFE膜材在该阶段 受力, 当外力消失以后, 膜材能完全恢复其原有形状即膜 材的内部纱线未发生塑性变形; 膜材的塑性阶段表现为弹 性阶段之后若撤去该部分外力, 膜材不能恢复其原有形 状, 说明其内部纱线已经产生塑性形变; 而膜材到达破坏 阶段时不断承受施加的双向荷载, 内部纱线逐渐断裂滑 移, 从而导致整体膜材的损坏。两种中心孔径膜材在拉伸 直至断裂过程中均表现为弹性阶段以及破坏阶段膜材孔 周最大应变快速增长, 而在塑性阶段膜材孔周最大应变呈 现缓慢增长的趋势, 然而在含孔膜材的弹性阶段, 其应变 指标演化曲线基本重合, 也就是说对于含孔膜材, 其弹性 阶段的应变值跟孔径的大小没有直接关系, 只跟膜材自身 材料的本构关系有关, 而两种孔径膜材过了弹性阶段到达 塑性阶段以后, 中心孔径起到了直接影响, $4 \mathrm{~mm}$ 孔膜材的

$$
Y 1=\left\{\begin{array}{l}
9.08 x+0.338(\text { Oa 阶段 }) \\
1.83 x+7.644(a b \text { 阶段 }) \\
2.87 x+2.946 \text { (bc 阶段) }
\end{array}\right.
$$

\section{2. 含孔膜材应变集中系数分析}

根据应力集中的定义: 对于含孔构件在受到拉伸荷载 时, 孔边沿受拉方向的另一个方向会产生应力集中, 定义 孔口两侧最大应力即峰值应力 $\sigma_{\text {max }}$, 将孔边峰值应力 $\sigma_{\text {max }}$ 与基准应力 $\sigma_{0}$ 的比值定义为应力集中系数。同理, 由应力 集中系数我们可以定义应变集中系数: 根据含孔膜材左右 两侧应变集中带, 取最大应变孔两侧的纵向应变 $\varepsilon_{y}$ 以及膜 材远场处的应变 $\varepsilon_{\mathrm{y}}{ }^{\infty}$ 的比值定义为应变集中系数。
孔周最大应变在拉伸过程中表现出的应变大于 $2 \mathrm{~mm}$ 孔径 膜材, 这是由于内部孔的存在, 孔径越大, 原本圆孔处的 纱线被破坏的越多, 导致膜材在塑性阶段受拉伸力以后, 相对于 $2 \mathrm{~mm}$ 孔径的膜材不能很好的控制其内部纱线变形, 其应变就会略大于 $2 \mathrm{~mm}$ 中心孔膜材, 当两种孔径膜材均达 到破坏阶段以后, 应变快速增长直至膜材完全断裂, 且最 终断裂时 $4 \mathrm{~mm}$ 孔径的孔周最大应变大于 $2 \mathrm{~mm}$ 孔径, 然而其 断裂力却远远小于 $2 \mathrm{~mm}$ 孔径中心膜材, 说明膜材孔的存在 会催化膜材的断裂, 且孔径越大, 膜材孔边应变集中更加 明显, 膜材所能承受的极限荷载越小, 当含孔膜材经历一 个孔周最大应变由快速上升到缓慢上升再到快速上升的 阶段时, 表示含孔膜材即将破坏, 同时证实应变场指标可 以作为含孔膜材破坏前兆性指标。将这两种中心孔径膜材 三个阶段的应变曲线分别拟合成下式 (将各阶段近视为直 线), $2 \mathrm{~mm}$ 孔径为 $Y_{1}, 4 \mathrm{~mm}$ 孔径为 $Y_{2}, Y_{1} 、 Y_{2}$ 所代表数值 均为百分制数值, 即可根据动态信号测试系统所测得的双 向拉伸力值大致预知两种中心孔径膜材在整个拉伸过程 中孔周最大应变。

$Y 2=\left\{\begin{array}{l}9.82 x+0.271(O A \text { 阶段 }) \\ 2.51 x+8.395(A B \text { 阶段 }) \\ 11.995 x-29.597(B C \text { 介段 })\end{array}\right.$

$$
K=\frac{\varepsilon y}{\varepsilon y \infty}
$$

其中膜材远场处的应变 $\varepsilon_{y}^{\infty}$, 由于PTFE膜材为正交各向 异性材料, 其在拉伸时表现出应变分布的随机性, 单取某 点值计算结果误差较大, 不具备参考价值, 故本文选取散 斑构造区域的应变平均值作为其远场处的应变 $\varepsilon_{\mathrm{y}}{ }^{\infty}$, 计算得 到含孔PTFE膜材在双向拉伸下的应变集中系数K如表2。

表2 含孔膜材应变集中系数 $\mathrm{K}$ 。

\begin{tabular}{llll}
\hline 分类 & $\boldsymbol{\varepsilon}_{\mathbf{y}}(\mathbf{\%})$ & $\boldsymbol{\varepsilon}_{\mathbf{y}}^{\infty} \mathbf{( \% )}$ & $\mathbf{K}($ 无量纲) \\
\hline $2 \mathrm{~mm}$ 中心孔 & 6.942 & 3.054 & 2.273 \\
$4 \mathrm{~mm}$ 中心孔 & 17.722 & 4.528 & 3.914 \\
\hline
\end{tabular}

由表 2 含孔膜材应变集中系数K计算结果可以发现, 在 双向拉伸下, PTFE含孔膜材直径的大小对其应变集中系 数起到了直接影响, 且膜材孔径越大, 应变集中系数越大。 对于 $2 \mathrm{~mm}$ 孔双向拉伸下应变集中系数比较小, 这种孔在膜 材上相对而言比较安全, 而对于大孔径的受损 膜材应该 注意到其孔边较大的应变集中系数, 说明膜材受到损伤越 大时, 受拉状态下孔边应变集中现象更为明显, 更容易达 到极限荷载而发生撕裂破坏。

\section{ABAQUS模拟结果与分析}

\section{1. 模型建立与处理}

本文双向拉伸试样模型采用二维Shell矩形模型, 在 Part模块里建立 $100 \mathrm{~mm} \times 30 \mathrm{~mm}$ 的矩形模型, 并根据实验方
案对各矩形模型进行掏孔处理; 在Property模块中设置弹 性材料属性, 由于PTFE膜材为正交各向异性材料, 根据 试验数值设置其弹性模量以及泊松比, 同时设置柔性损伤 以及损伤演化; 根据膜材试样所测得拉伸力数据对各种含 孔模型进行载荷加载, 拉伸两端均只有一个自由度; 网络 划分技术采用结构化划分技术, 将膜材划分为完全由三角 形组成的网格, 提交计算进行后处理查看各种含孔模型在 拉伸直至断裂过程的应变云图。

\section{2. 模拟结果分析}

根据实验相对应的各种含孔模型的模拟, 分析得到各 组在拉伸直至断裂时的应变云图如图7所示。 

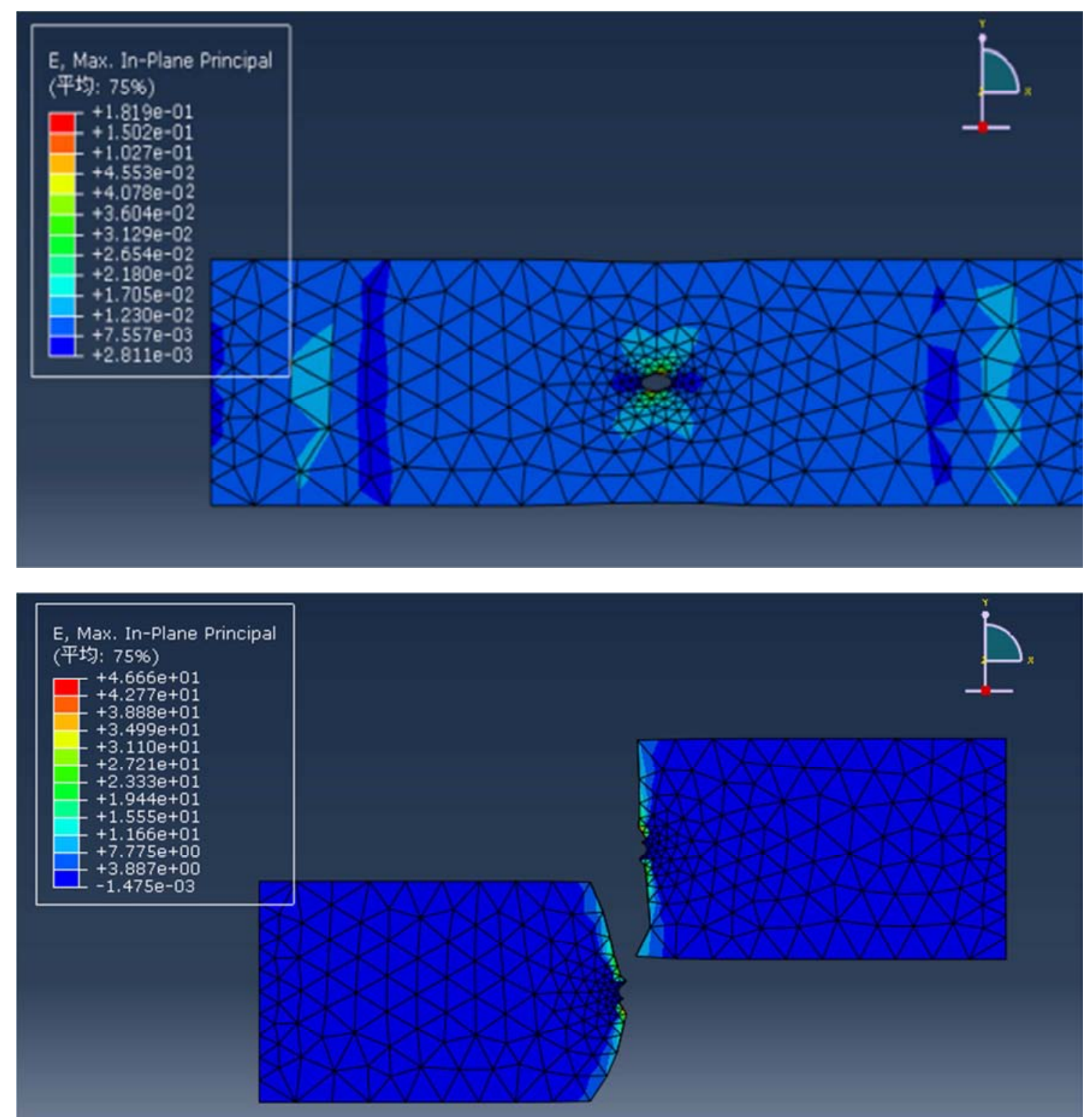

(a)2mm中心孔模型

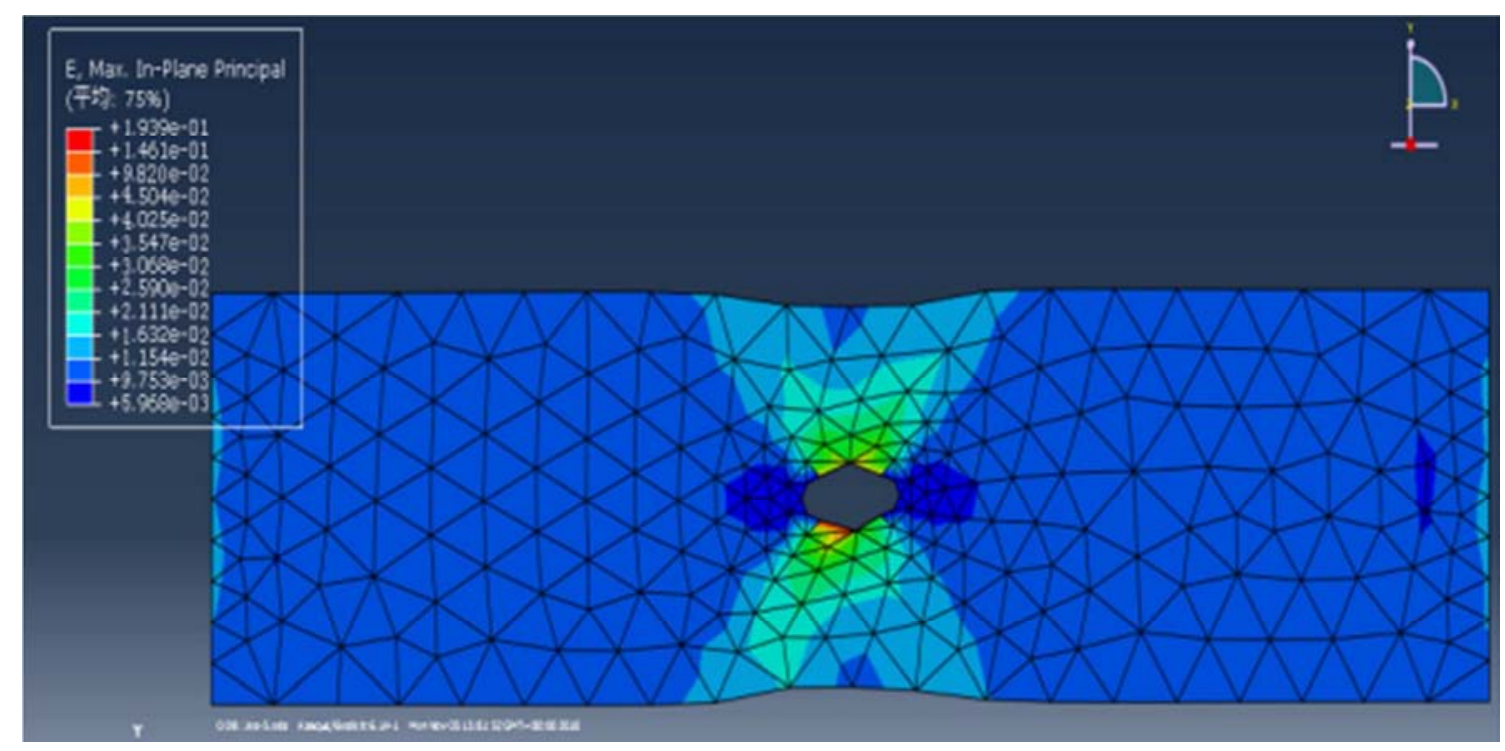




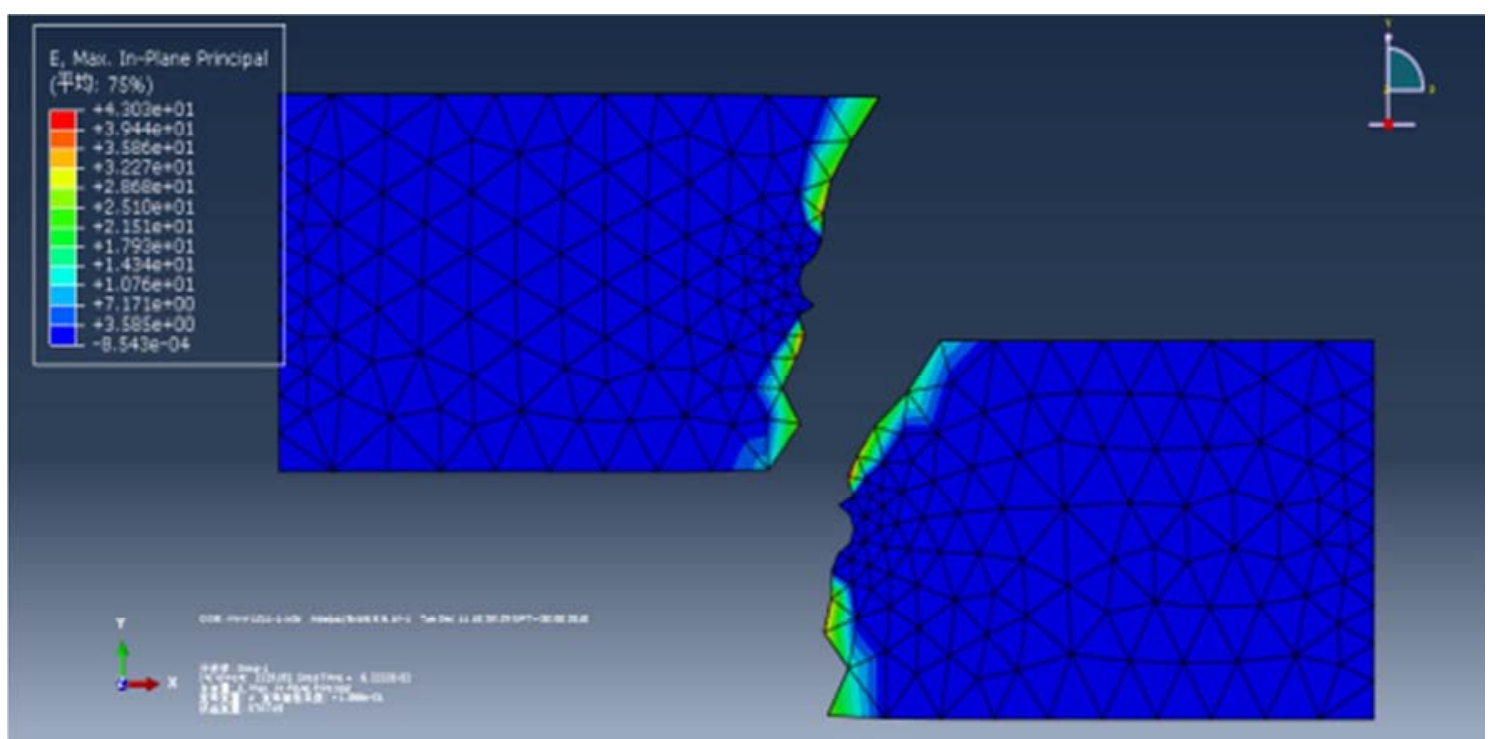

(b) $4 \mathrm{~mm}$ 中心孔模型

图7 各含孔模型拉伸断裂前后分析步应变云图。

由图 $2 \mathrm{~mm}$ 与 $4 \mathrm{~mm}$ 中心孔模型双向拉伸断裂云图可以 看出, 在模型断裂之前这两种孔径膜材均在孔周形成了较 严重的应变集中现象，且应变分布呈区域性集中，然而在 远离孔周处, $2 \mathrm{~mm}$ 孔径膜材表现出的应变集中区域相对 $4 \mathrm{~mm}$ 孔径膜材较小, 这说明当膜材上存在小直径孔时, 对 膜材的影响比较小; 由于膜材为正交各向异性材料, 故在 模型其他区域应变演化云图上会呈现出应变交替分布的 现象, 由模拟结果可知, 在拉伸断裂前一个分析步 $2 \mathrm{~mm}$ 和 $4 \mathrm{~mm}$ 孔径模型孔周最大应变分别为 0.182 和 $0.194,4 \mathrm{~mm}$ 孔 径模型应变略大于 $2 \mathrm{~mm}$ 孔径模型, 这也证实了膜材孔径的 大小影响着张拉膜材全场应变, 且孔径越大应变越大; 随 着双向拉伸力的持续加载, 中心孔逐渐发生变异直至达到 峰值应变模型发生断裂, 由 $2 \mathrm{~mm}$ 及 $4 \mathrm{~mm}$ 中心孔模型断裂瞬 时分析步的应变云图可以发现, 两种不同孔径中心孔模型

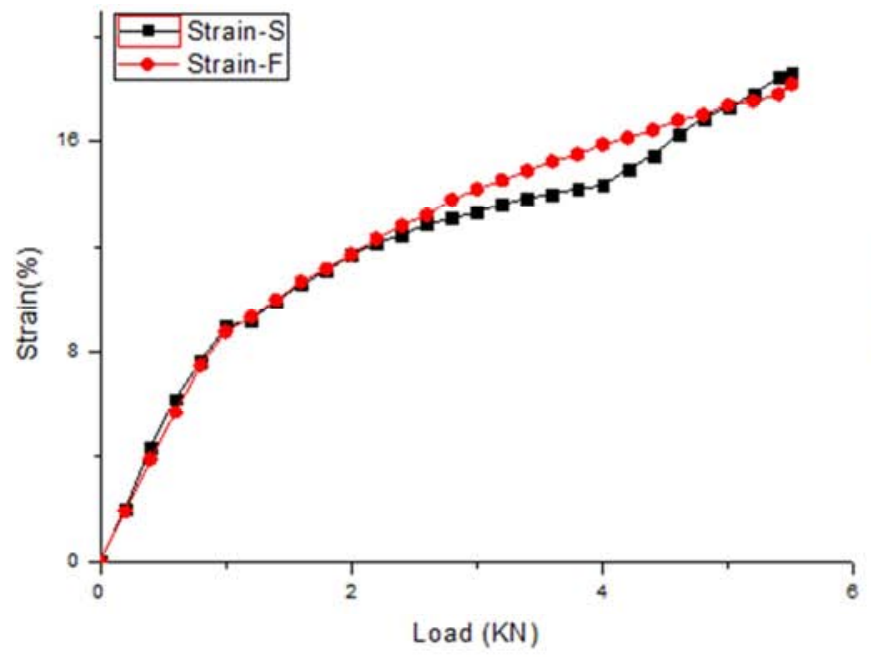

(a) $2 m m$ 中心孔
在断裂后膜材上绝大部分区域均呈应变负值, 即膜材在断 裂瞬时发生了收缩现象, 这是由于膜材在拉伸过程中一直 处于受拉紧绷状态, 而在模型达到应变峰值断裂瞬时张紧 的结构会立马丧失弹力向内收缩, 模型断裂的方向均垂直 于模型拉伸方向, 从中心孔出发沿着膜材纬向形成一条贯 穿膜材宽的裂纹而发生拉断。

\section{3. 实验与模拟分析对比}

为了更加直观地了解模拟结果, 本文将各组含孔模型 从拉伸直至断裂各分析步所代表的荷载值以及其对应的 两组含孔膜材 A、B点处的孔周最大应变模拟值对比实验 结果绘制成如图8所示图线。

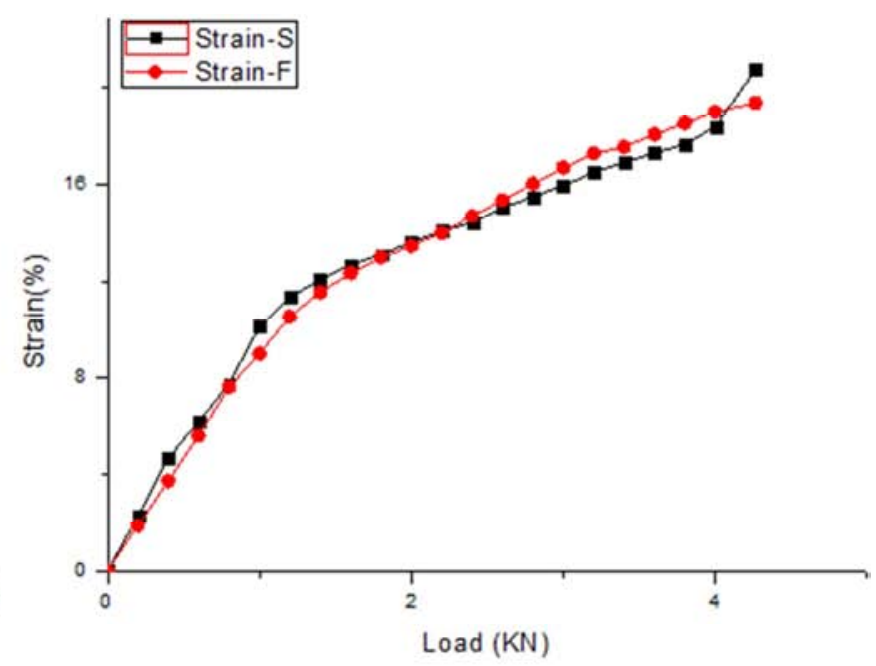

(b) $4 \mathrm{~mm}$ 中心孔

图8 不同孔径数值模拟与实验结果对比。 
由图8所示含不同孔径膜材在ABAQUS模拟以及实验 中双向拉伸力与孔周最大应变对比图可以发现, 模拟过程 中PTFE膜材在拉伸直至断裂的拉伸力--孔周最大应变曲 线图没有像实验过程中表现出明显的三阶段性质, 而是呈 现出在双向拉伸初期随着荷载的增长应变快速增长的现 象, 当拉伸力达到 $2 \mathrm{KN}$ 以后, 随着荷载的增长孔周最大应
变缓慢增长直至模型断裂。由上面两组图线可以看出 ABAQUS模拟结果与实验结果吻合性较好, 各组含孔膜材 在拉伸过程中模拟与实验应变值上升趋势基本一致。各组 模型及试验样品断裂时孔周应变集中系数 $\mathrm{K}$ 对比分析如表 3 , 在断裂时孔周应变集中系数的模拟值与试验值误差均 不超过 $10 \%$, 故上述模拟方法具有相对可靠性。

表3 模拟及试验断裂孔周应变集中系数 $\mathrm{K}$ 。

\begin{tabular}{|c|c|c|c|}
\hline & ABAQUS模拟值 & 试验值 & （|模拟值-试验值|/试验值）×100\% \\
\hline 2mm中心孔 & 2.098 & 2.273 & $7.7 \%$ \\
\hline 4mm中心孔 & 3.586 & 3.914 & $8.4 \%$ \\
\hline
\end{tabular}

\section{5. 结论}

基于数字图像散斑技术, 通过单轴双向拉伸试验研究 了中心含不同直径孔PTFE膜材在拉伸全过程的全场应变 以及膜材断裂时的孔周极限应变, 得到膜材在双向拉伸过 程中呈现出了三阶段性质: 弹性阶段、塑性阶段以及破坏 阶段; 含孔膜材拉伸断裂时的孔周最大应变以及膜材的极 限承载力与膜材自身的材料特性以及孔径大小有关, 孔径 越大其孔边应变集中系数越大, 所能承受极限荷载越小, 膜材越易损坏。

通过ABAQUS数值模拟与实验进行对比, 验证了含孔 PTFE膜材在拉伸直至断裂时的应变特性, 可以通过模拟 预先得知含孔PTFE膜材在拉伸全过程的全场应变变化以 及孔周最大应变情况。本文未涉及切口、孔群等其它形式 的损伤对膜材力学性能的影响, 将在后续工作中做更加深 入地研究。

\section{参考文献}

[1] 张营营, 张其林. 涂层织物类建筑膜材料的力学性能 $[\mathrm{M}]$. 中国矿业大学, 2013。

[2] Andrzej Ambroziak, Paweł Kłosowski. Mechanical properties for preliminary design of structures made from PVC coated fabric[J]. Construction and Building Materials, 2014,50:74-81.

[3] 黄祺合,芦继忠,陶富录,陈学良,周汝贵. 大跨度膜结构屋面 安装施工技术 $[\mathrm{J}]$.结构施工，2018，40（9）：1541-1543。
[4] Bigaud D, Szostkiewicz C, Hamelin P. Tearing analysis for textile reinforced soft composites under mono-axial and bi-axial tensile stresses[J]. Composite Structures, 2003,62(2):129-137.

[5] 张营营, 张其林, 宋晓光. PTFE膜材力学性能及抗力不定 性分析[J].建筑材料学报, 2014，17（4）：726-733。

[6] 张营营, 许珊珊, 徐家豪. 聚四氟乙烯膜材黏弹性本构关 系[J].建筑结构学报，2016，37（6）：245-252。

[7] 马倩. 机织物撕裂破坏机理的有限元分析[J].东华大学, 2010。

[8] Lianxiang Yang, Yonghong Wang, Rongsheng Lu. Advanced Optical Methods for Whole Field Displacement and Strain Measurement[J]. Oakland University,2010.

[9] 张荵. 数字图像相关法及其在若干工程测试中的应用 $[\mathrm{J}]$. 华南理工大学, 2011。

[10] 谢学梁, 彭博, 汪文军, 王珏辉, 刘旨阳. 结构健康监测 系统的设计与实现 [J]. 电子测试, 2018,22（025）：60-62。

[11] 高新京, 吴明超. 膜结构工程技术与应用 $[\mathrm{M}]$. 北京: 机械工 业出版社，2010：23-25。

[12] 陈新忠, 曹远威, 刘世伟, 毛仲敏, 张吉雄. 基于数字图 像的非接触式泊松比测试方法 [J]. 煤炭学报, 2014,39 (S2) :366-371。

[13] 章辉, 郑宇英. 膜结构用涂层织物拉伸与撕裂性能测试方 法及关系研究 [J].纺织标准与质量, 2009, 4：34-39。

[14] 汪泽幸, 何斌, 陈妍, 李洪登. 损伤条件下聚氯乙烯涂层 膜结构材料拉伸蠕变特性 [J]. 纺织学报, 2017, 38 (10):57-64。 